\title{
EFECTO DE LA APLICACIÓN DE AUXINAS Y CALCIO A LAS ÚLTIMAS MANOS DEL RACIMO DEL BANANO PARA MEJORAR CALIBRACIÓN Y LARGO DE DEDOS DE LA FRUTA
}

\author{
EFFECT OF THE APPLICATION OF AUXINS AND CALCIUM TO THE LAST \\ HANDS OF THE BANANA CLUSTER TO IMPROVE FRUIT FINGERTIP AND \\ LENGTH CALIBRATION
}

\author{
VALERIANO BUSTAMANTE GARCÍA', DEYNIS GÓMEZ TOMALÁ² \\ 1 Universidad de Guayaquil. valeriano.bustamante@ug.edu.ec \\ 2 Universidad de Guayaquil.deynisgt_94@hotmail.com
}

RESUMEN

La presente investigación se realizó en el Recinto San Carlos del Cantón Balao provincia del Guayas. Se evaluó el efecto de la aplicación de auxinas y calcio a las últimas manos del racimo de banano para mejorar la calibración y largo de los dedos. El objetivo fue el de generar tecnologías mediante la aplicación de bioestimulantes para mejorar el calibre de la fruta, el largo de dedos y en consecuencia la calidad del racimo, mediante la aplicación del bioestimulante (auxinas) más emulsionante (calcio) para mejorar la calidad de la fruta. Los tratamientos fueron, tres aplicaciones de Basfoliar Kelp (Auxinas) + Basfoliar Calcio (Ca) y un testigo convencional, fueron analizados en un diseño de bloques completamente al azar con arreglo grupal con 4 repeticiones y la comparación de las medias de acuerdo a la prueba de Tukey al $0.05 \%$ de probabilidades.

El ensayo se lo realizo en un tiempo mínimo de 12 semanas en campo y se registraron los datos en la cosecha, en la empacadora. Se trabajó con 4 tratamientos, tomando 20 racimos por tratamientos, es decir 80 racimos por semana cintas en todo el experimento, con las repeticiones durante 2 semanas (dos cintas de tratamiento). Se realizó la primera atomización, cuando la bellota tenía 4 manos expuestas (a los 4 días de enfundada aproximadamente después del inicio de floración). La segunda aplicación se llevó a efecto, cuando las manos están expuestas en su totalidad (10 días después de floración). Se realizó falsa + 2 en todo el tratamiento. Los resultados de auxinas (Basfoliar kelp) + calcio (basfoliar calcio) realizada a los 4 días y a los 10 días después de las aplicaciones mostraron un alargamiento y grosor de la fruta en los tratamientos mientras que en el testigo convencional los dedos eran más cortos y menos gruesos, siendo el tratamiento 2 (100cc Basfoliar kelp +100cc de Basfoliar calcio) el que obtuvo mejores resultados en calidad de fruta.

PALABRAS CLAVE: calidad de frutos, bioestimulante auxinas, emulsionantes calcio banano
ABSTRACT

This following research study was conducted in San Carlos Precinct of the Canton Balao, province of Guayas. The effect of the application of biostimulant (auxins) plus emulsifier (calcium) to the last clutters of the banana bunchs was evaluated to improve the calibration and length of the fingers, whose objective was to generate technologies through its application to escalate the size of the fruit, the length of the fingers and consequently the quality of the bunch, to finally improve the quality of the fruit. The treatments were, three applications of Basfoliar Kelp (Auxinas) + Basfoliar Calcio (Ca) and a conventional control, were analyzed in a fully random block design with an arrangement group of four repetitions and the comparison of the means according to the test of Tukey at $0.05 \%$ probability.

The test was carried out in a minimum time of 12 weeks in the field and the data were recorded during the harvest, in the packing house. It was worked with 4 treatments, taking 20 clusters per treatment, which means 80 clusters per week tapes throughout the experiment, with repetitions for 2 weeks (two treatment tapes). The first dash was applied when the acorn had 4 hands (after 4 days of sheathing approximately after the beginning of flowering). The second application took effect, when the hands were fully exposed ( 10 days after flowering). It was made false +2 in all treatments. The results of auxins (Basfoliar kelp) + calcium (basfoliar calcium) performed at 4 days and 10 days after the applications showed an elongation and thickness of the fruit, while with the conventional control the fingers were shorter and less thick, being the second treatment (100cc Basfoliar kelp + $100 \mathrm{cc}$ of Basfoliar calcium), the one that obtained better results in terms of quality.

KEYWORDS: fruit quality, auxin biostimulants, banana calcium emulsifiers. 
INTRODUCCIÓN

La actividad bananera en el Ecuador desde hace sesenta años ha tenido y tiene un peso importante en el desarrollo del país, tanto desde el punto de vista económico como social. En lo económico por su participación en el PIB y en la generación de divisas y en lo social por las fuentes de empleo que genera y más aún por su peso importante en determinadas regiones de la costa ecuatoriana (James, 2009).

Ecuador cuenta con las condiciones idóneas para cultivar un banano de excelencia, se mantiene como el primer exportador, y el banano de mejor calidad en el mundo, el $30 \%$ de la oferta mundial de banano proviene de Ecuador, representando el 15\% del total de las exportaciones y se incrementó en un $12 \%$ entre enero y octubre de 2017 y el mismo período de 2016. Según datos del banco central del Ecuador (BCE), en dicho lapso de 2017 se han vendido un total de 5.333 toneladas de la fruta por $\$ 2.490$ millones. El principal destino del producto ecuatoriano fue la Unión Europea, con una participación del 35\%; seguido de Rusia con el 24\%; y en tercer lugar, Estados Unidos con el 18\%. (El Telégrafo, E. 2018)

El cultivo de banano requiere de una serie de labores, tanto en el campo como en la planta empacadora, cada una de estas labores cuando son mal ejecutadas, causan enormes pérdidas de producción (Aboboreira, 1994).

El desmane o deschive es una práctica cultural que se realiza durante la protección de la fruta cuando los frutos están colocados en dirección hacia abajo sin usar herramienta alguna, simplemente con la mano, consiste en eliminar ocasionalmente la última mano o falsa mano y una o las dos siguientes que se estime que no llegaran adquirir el tamaño mínimo requerido, favoreciendo al desarrollo de las restantes para aumentar el largo, tamaño de los dedos y también el peso del racimo. Esto se la conoce como falsa uno, dos, tres, o cuatro, dependiendo del número de manos que se eliminen (Alban, 2014).

El tamaño de la fruta es característica importante para los mercados especializados de banano, y para mejorarla se ha recurrido a la eliminación selectiva de manos del racimo, con base en el mayor tamaño de las manos proximales ((Aristasabal, 2008). Sin embargo, aunque el desmane mejora el peso y tamaño de los dedos, el peso total se reduce. En este punto surgió la interrogante de si era posible aumentar el tamaño de los dedos recurriendo a prácticas adicionales en racimos sometidos a desmane y cuando se aplica en racimos formados de banano Gran Nain, los frutos se mantienen más verdes y firmes durante dos semanas. En variedades enanas de plátanos y bananos aumenta la elongación celular en los tejidos, incrementa el tamaño de hojas y tallos, especialmente, de los frutos; no obstante, esta última característica no se ha cuantificado (Aristasabal, 2008).

La importancia de la calidad del banano producido en Ecuador al ser producto de exportación, está sujeto a los requerimientos y exigencias de calidad demandados en los principales mercados mundiales; por esta razón el banano de exportación debe de cumplir una serie de

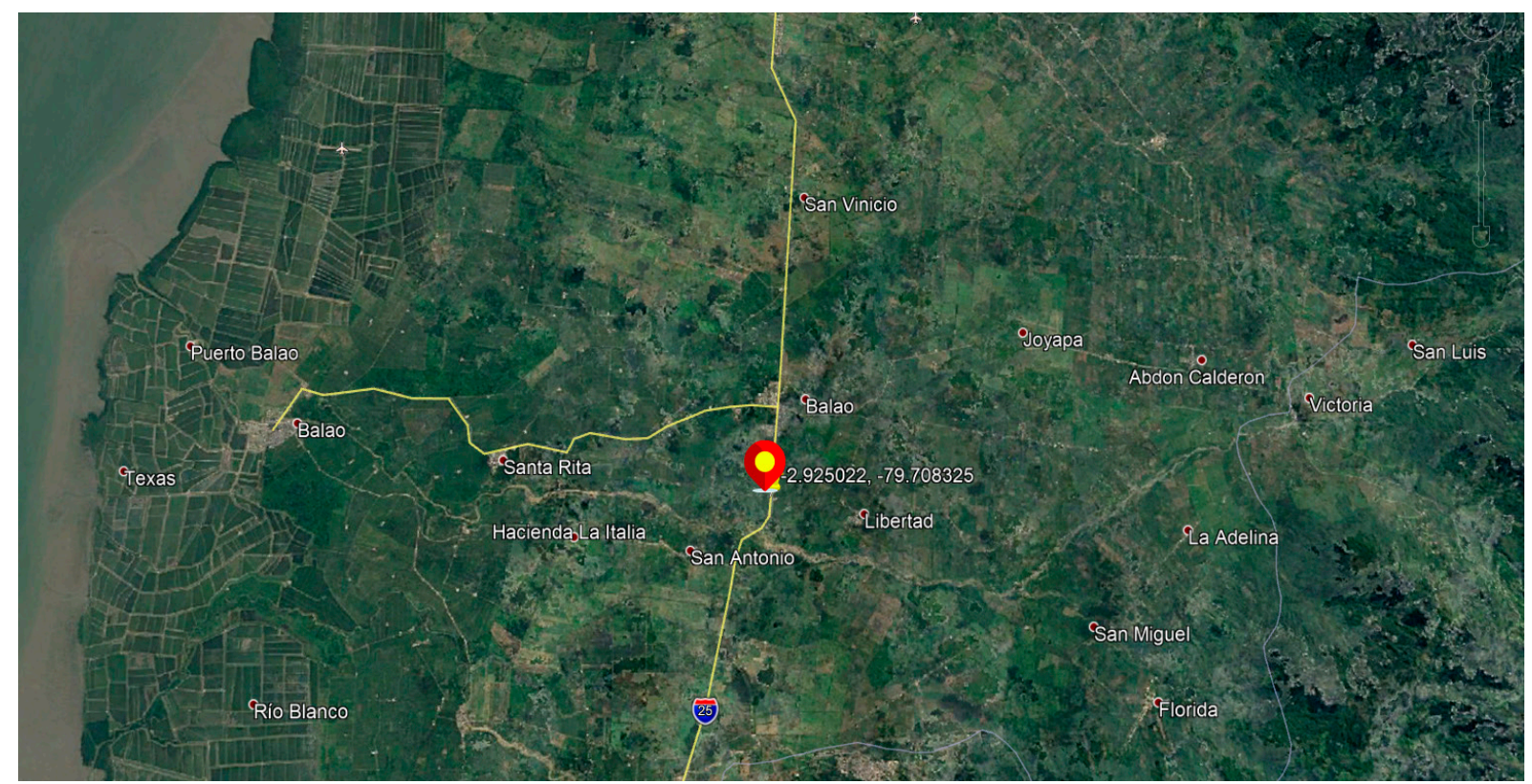

Figura 1: Mapa de la localización de la investigación 
requerimientos en su presentación tales como: curvatura, tamaño, grosor, peso, sin cicatrices, ni manchas, requisitos que debe cumplir, caso contrario es desechado y clasificado como rechazo lo que genera una pérdida económica para la organización (Pardo \& Novillo, 2016). La presente investigación tuvo como objetivo el generar tecnologías mediante la aplicación de bioestimulantes, auxinas y calcio para mejorar el calibre de la fruta, el largo de dedos y en consecuencia la calidad del racimo.

\section{LOCALIZACIÓN}

La presente investigación se realizó en la hacienda "Italia" propiedad del Sr. Kleber Pita ubicada en el recinto San Carlos del cantón Balao, provincia del Guayas cuyas coordenadas son $2^{\circ} 55^{\prime} 30.4^{\prime \prime}$ al sur y $79^{\circ} 42^{\prime} 03.0$ al oeste (ver Fig. 1)

En la tabla 1 se muestran las características del clima y suelos del lugar donde se realizó la investigación.

TABLA 1. CARACTERÍSTICAS METEREOLÓGICAS DEL CANTÓN BALAO

\begin{tabular}{lc}
\hline Clima & Tropical- sabana \\
\hline Altura & $10 \mathrm{~m} . s . n \cdot m$ \\
\hline Temperatura & $15^{\circ} \mathrm{C}$ a $34^{\circ} \mathrm{C}$ \\
\hline Humedad relativa & $72 \mathrm{y} 80 \%$ \\
\hline Precipitación anual & $1000 \mathrm{~mm}$ \\
\hline Vientos & 25 a $30 \mathrm{k} / \mathrm{h}$ \\
\hline Suelos & Franco- arcillo- arenoso \\
\hline Importaciones & 4,026
\end{tabular}

Fuente: Datos obtenido del Gobierno Autónomo Descentralizado. Municipal de Balao.

\section{CROQUIS DE CAMPO}

Área del experimento realizado, sus dos caras del lote 5

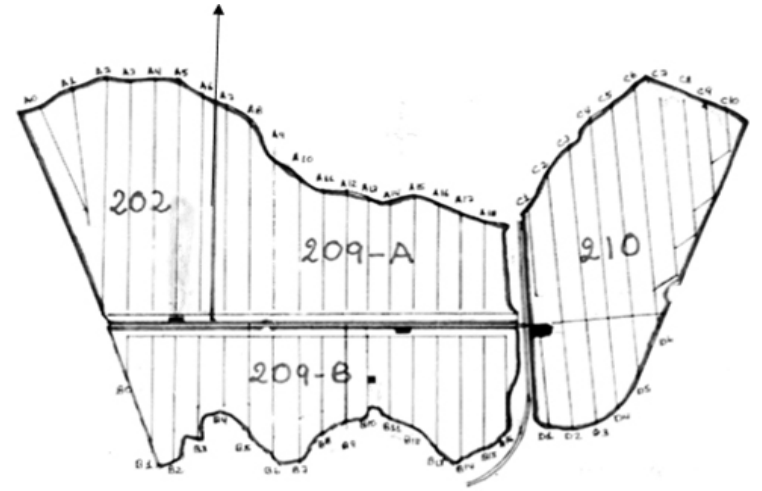

METODOLOGÍA

Los factores en estudio fueron dos niveles (4- 10 días) del bioestimulante regulador de crecimiento (Basfoliar kelp LS), así como dos niveles de calcio emulsionable (Basfoliar $\mathrm{Ca}$ ), aplicadas a las últimas manos del racimo, cuando el ra- cimo tenga no más de 10 días de desarrollo, los tratamientos estudiados, aplicaciones y dosis se presentan en la tabla 2.

\section{TABLA 2. TRATAMIENTOS ESTUDIADOS}

\begin{tabular}{|c|c|c|c|}
\hline TRATAMIENTOS & $\begin{array}{c}\text { 1ERA } \\
\text { APLICACIÓN }\end{array}$ & $\begin{array}{c}\text { 2DA } \\
\text { APLICACIÓN }\end{array}$ & DOSIS \\
\hline $\begin{array}{l}\text { T1= Regulador de } \\
\text { crecimiento (Basfoliar } \\
\text { Kelp) + calcio emulsio- } \\
\text { nable (Basfoliar Ca). }\end{array}$ & 4 días & 10 días & $\begin{array}{c}75 c c+75 c c \\
/ 1 \text { L agua }\end{array}$ \\
\hline $\begin{array}{l}\text { T2= Regulador de } \\
\text { crecimiento (Basfoliar } \\
\text { Kelp) + calcio emulsio- } \\
\text { nable (Basfoliar Ca). }\end{array}$ & 4 días & 10 días & $\begin{array}{l}100 \text { cc +100 } \\
\text { cc / } 1 \text { L agua }\end{array}$ \\
\hline $\begin{array}{l}\text { T3= Regulador de } \\
\text { crecimiento (Basfoliar } \\
\text { Kelp ) + calcio } \\
\text { emulsionable (Basfoliar } \\
\text { Ca). }\end{array}$ & 4 días & 10 días & $\begin{array}{l}12,5 \text { cc }+12,5 \\
\text { cc / } 1 \text { I agua }\end{array}$ \\
\hline $\begin{array}{l}\text { T4=Testigo convencional } \\
(\text { falsa }+2)\end{array}$ & s. t & st & s.t \\
\hline
\end{tabular}

s.t= sin tratamientos

El ensayo se lo realizó en un tiempo mínimo de 12 semanas en campo y se registraron los datos de la cosecha, en la empacadora. Se trabajó con 4 tratamientos.

Se tomaron 20 racimos por tratamientos, es decir 80 racimos por semana cintas en todo el experimento.

Se realizaron las repeticiones durante 2 semanas (dos cintas de tratamiento).

Se procedió a realizar la primera atomización, cuando la bellota tuvo 4 manos expuestas (a los 4 días de enfundada aproximadamente después del inicio de floración).

La segunda aplicación se llevó a efecto, cuando las manos estuvieron expuestas en su totalidad (10 días después de floración).

Se realizó falsa +2 en todos los tratamientos.

Se utilizó el diseño de Bloques completamente al azar con arreglo grupal; con 4 tratamientos y 4 repeticiones. Todas las variables fueron sometidas al análisis de varianza y para determinar la diferencia estadísticas entre las medidas de los tratamientos se empleó la Prueba de Tukey al $5 \%$ de significancia. Tabla 3

\section{TABLA 3. ANÁLISIS DE VARIANZA}

\begin{tabular}{lc|}
\hline FUENTE DE VARIACIÓN & GRADOS DE LIBERTAD \\
\hline Repetición $(\mathrm{t}-1)$ & 3 \\
\hline tratamientos $(\mathrm{r}-1)$ & 7 \\
\hline Grupo cinta verde $(\mathrm{gcv}-1)$ & 3 \\
\hline Grupo cinta azul $(\mathrm{gca}-1)$ & 3 \\
\hline Entre grupos (grupos -1$)$ & 3
\end{tabular}




\begin{tabular}{|lc|}
\hline FUENTE DE VARIACIÓN & GRADOS DE LIBERTAD \\
\hline Error experimental $(\mathrm{t}-1 \mathrm{x}$ r-1) & 21 \\
\hline Total (t-1) & 31 \\
\hline
\end{tabular}

\section{RESULTADOS}

En la tabla 4, el peso de los racimos en $\mathrm{kg}$, se observa que dentro del grupo de la cinta verde el mayor promedio se obtiene con el tratamiento de Basf foliar kelp + Basf foliar Ca (100 + $100 \mathrm{cc} / \mathrm{ha})$ con un valor de $36,06 \mathrm{~kg} / \mathrm{racimo}$ superior estadísticamente a los tres tratamientos restantes; en el grupo de la cinta azul todos los promedios de los tratamientos fueron iguales estadísticamente.

\section{TABLA 4. PESO DE LOS RACIMOS POR TRATAMIENTOS EN KG}

\begin{tabular}{|c|c|c|c|c|c|}
\hline DosIs & $\begin{array}{c}75+75 C C / \\
\text { LAGUA }\end{array}$ & $\begin{array}{c}100+100 \mathrm{CC} / \\
\text { LABUA }\end{array}$ & $\begin{array}{c}12,5+12,5 C C / \\
\text { LAGUA }\end{array}$ & SIN APLICACIÓN & MEDIA \\
\hline Grupo cinta verde & $30,36 \mathrm{~b}$ & 36,06 a & $28,85 \mathrm{bb}$ & $28,85 \mathrm{~b}$ & 31.03 \\
\hline Grupo cinta azul & 28,67 a & $30,53 \mathrm{AA}$ & $28,40 \mathrm{~A}$ & $28,70 \mathrm{AA}$ & 29.07 \\
\hline C.V $(\%)$ & 9.39 & & & & \\
\hline
\end{tabular}

En la Fig. 2 se muestra la comparación entre los promedios de los dos grupos de cintas para la variable de peso de racimos por tratamientos expresados en $\mathrm{kg}$.

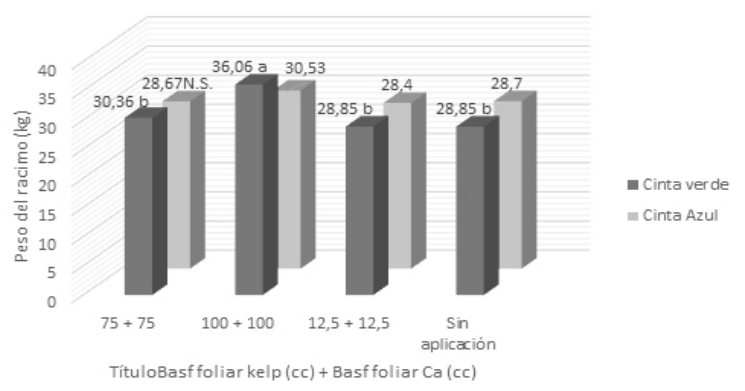

Figura 2: Comparación entre promedios de dos grupos de cinta para la variable peso de racimo $(\mathrm{kg})$

En la tabla 5, se observa el largo de los dedos de la última mano, que dentro del grupo de la cinta verde el mayor promedio se obtiene con el tratamiento de Basf foliar kelp + Basf foliar Ca $(100+100 \mathrm{cc} / \mathrm{ha})$ con un valor de 8.98 pulgadas /racimo superior estadísticamente a los tres tratamientos restantes; en el grupo de la cinta azul todos los promedios de los tratamientos fueron iguales estadísticamente.

TABLA 5. LONGITUD DE DEDOS DE LAS ÚLTIMAS MANOS DEL RACIMO (PULGADAS)

\begin{tabular}{|c|c|c|c|c|c|}
\hline Dosis & $\begin{array}{c}75+75 C C / 1 \\
\text { LAGUA }\end{array}$ & $\begin{array}{c}100+100 C C / \\
\text { LAGUA }\end{array}$ & $\begin{array}{c}12,5+12,5 C C / \\
\text { LAGUA }\end{array}$ & SINAPLICAcIÓN & MEDIA \\
\hline Grupo cinta verde & $8,53 \mathrm{bb}$ & $8,98 \mathrm{a}$ & $8,36 \mathrm{bb}$ & 8,166 & 8,5 \\
\hline Grupo cinta azul & 8,38 bb & $8,76 \mathrm{a}$ & $8,17 \mathrm{bcc}$ & $8,01 c$ & 8,33 \\
\hline
\end{tabular}

C.V (\%) 2,06
En la fig. 3 se observan los promedios de los dos grupos de cinta para la variable largo de dedos de las últimas manos expresado en pulgadas.

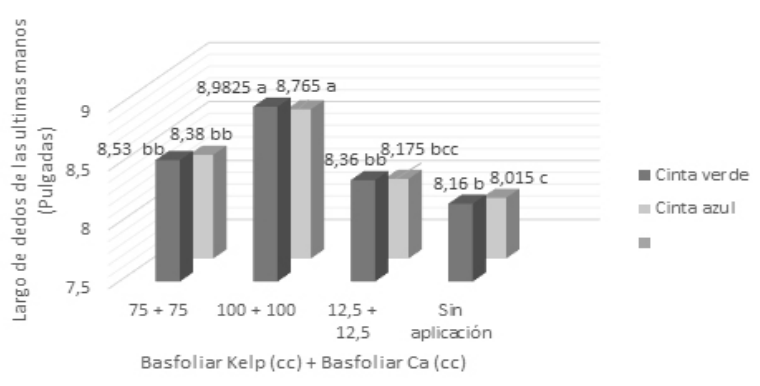

Figura 3: Comparación entre promedios de dos grupos de cinta para la variable largo de dedos de las últimas manos (pulgadas)

Al efectuar la comparación entre los promedios generales de los dos grupos de cinta, con el grupo de la cinta verde se obtuvo un promedio de 8.5 pulgadas/racimo diferente estadísticamente al grupo de la cinta azul cuyo valor fue de 8.33 pulgadas/racimo (tabla 6).

\section{TABLA 6. PROMEDIO GENERAL DE LA LONGITUD DE DEDOS DE LAS ÚLTIMAS MANOS DEL RACIMO}

DOSIS PROMEUIO

Grupo cinta verde

8,50 a

Grupo cinta azul

8,336

En la fig. 4 se muestran los promedios de largo de dedos de la últimas manos (pulgadas) de dos grupos de tratamientos con aspersiones directo al racimo de productos bioestimulante.

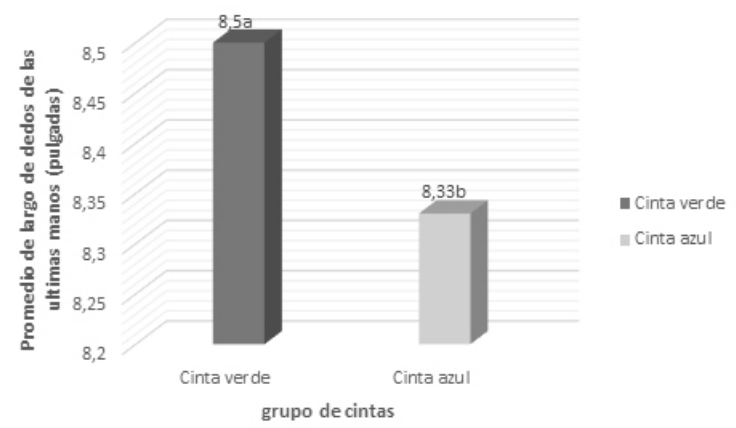

Figura 4: Comparación promedio de largo de dedos de la última mano (pulgadas) de dos grupos de tratamientos con aspersiones directo al racimo de productos bioestimulantes

En la tabla 7, se observa la calibración de las últimas manos del racimo (grados) que dentro del grupo de la cinta verde el mayor promedio se obtiene con el tratamiento de Basf foliar kelp + Basf foliar Ca $(100+100 \mathrm{cc} / \mathrm{ha})$ con un valor de 41.95 grados /racimo superior estadísticamente a los tres tratamientos restantes; en el grupo de 
la cinta azul todos los promedios de los tratamientos fueron iguales estadísticamente.

TABLA 7. CALIBRACIÓN POR tRATAMIENTOS DE LAS ÚlTIMAS MANOS DEL RACIMO (GRADOS)

\begin{tabular}{|c|c|c|c|c|c|}
\hline Dosis & 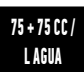 & $\begin{array}{c}100+1000 C / \\
\text { LAGUA }\end{array}$ & $\begin{array}{c}\text { 12,5+12,5cC/ } \\
\text { LAGUA }\end{array}$ & 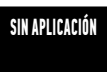 & MEDIA \\
\hline Grupo cinta verde & 41,75 аа & 41,95 аa & 40,90 a & 41,10 аа & 41.42 \\
\hline Grupo cinta azul & 40,80 aа & 41,05 аa & 40,75 аa & $40,20 \mathrm{a}$ & 40,7 \\
\hline
\end{tabular}

En la fig. 5 se detallan la comparación entre promedios de dos grupos de cinta para la variable calibración de las últimas manos (grados)

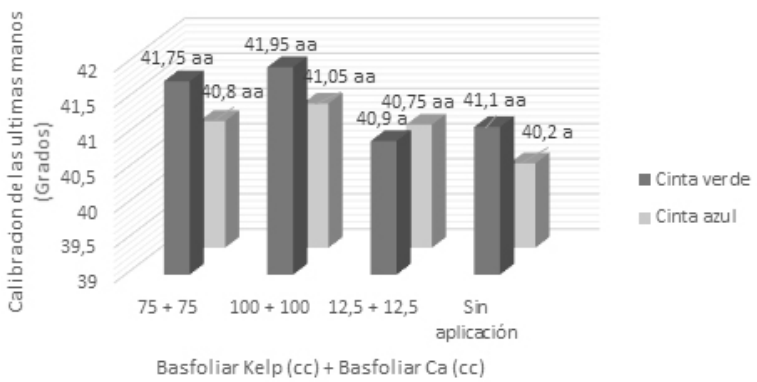

Figura 5: Comparación entre promedios de dos grupos de cinta para la variable calibración de las últimas manos (grados)

Al efectuar la comparación entre los promedios generales de los dos grupos de cinta, con el grupo de la cinta verde se obtuvo un promedio de 41.42 grados/racimo diferente estadísticamente al grupo de la cinta azul cuyo valor fue de 40.7 grados/ racimo (ver Tabla 8 )

tABLA 8. PROMEDIO GeNERAL DE LA CALIBRACIÓN DE LAS ÚLTIMAS MANOS DEL RACIMO

\begin{tabular}{lc} 
DOSIS & PROMEDIO \\
\hline Grupo cinta verde & $41,42 \mathrm{a}$ \\
Grupo cinta azul & $40,70 \mathrm{~b}$ \\
\hline
\end{tabular}

La comparación promedio de calibración de la últimas manos (pulgadas) de dos grupos de tratamientos con aspersiones directas al racimo de productos bioestimulantes se muestran en la fig. 5

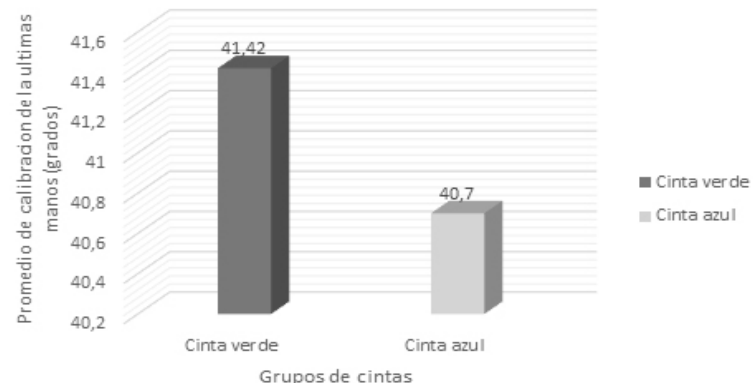

Figura 6: Comparación promedio de calibración de las últimas manos (pulgadas) de dos grupos de tratamientos con aspersiones directo al racimo de productos bioestimulantes.
En la tabla 9 se pueden apreciar los respectivos datos del ratio, donde la cinta color verde da como resultado del tratamiento T2 Basfoliar Kelp + Basfoliar Ca ( $100+100 \mathrm{cc} / \mathrm{ha})$ con un valor de 1.12 en la conversión de cajas por racimo ganando 2 cajas adicionales. Superior estadísticamente a los tratamientos restantes ; en el grupo de la cinta azul hubo una diferencia mínima del ratio de la cual bajo al 1.11

TABLA 9. RATIO DE LOS DIFERENTES TRATAMIENTOS

\begin{tabular}{|lcccc|}
\hline Dosis & $\begin{array}{c}75+75 \text { CC/L } \\
\text { AGUA }\end{array}$ & $\begin{array}{c}100+100 \text { CC/ } \\
\text { LAGUA }\end{array}$ & $\begin{array}{c}12,5+12,5 \text { CC/ } \\
\text { LAGUA }\end{array}$ & SIN APICACIÓN \\
\hline Grupo cinta verde & 1,05 & 1,12 & 1,03 & 1,02 \\
\hline Grupo cinta azul & 1,04 & 1,11 & 1,02 & 1,01 \\
\hline
\end{tabular}

La comparación promedio de calibración de la últimas manos (pulgadas) de dos grupos de tratamientos con aspersiones directo al racimo de productos bioestimulantes se muestran en la fig. 6.

\section{RATIO}

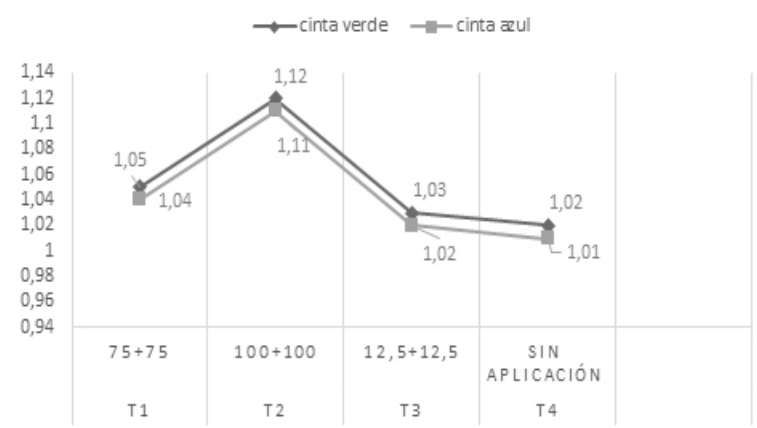

Figura 7: Comparación entre promedios de dos grupos de cinta para la observación del ratio

\section{DISCUSIÓN}

De acuerdo con los resultados obtenidos en la presente investigación, el T2 Basfoliar Kelp + Basfoliar Ca de $100 \mathrm{cc}+100 \mathrm{cc}$ en un litro de agua / ha,(bioestimulante); alcanzo el mayor peso del racimo, largo de dedos y calibración necesaria en las últimas manos, datos que se asemejan a lo que según (Lua, 2013).quien indica que los bioestimulantes contienen macro y micro nutrientes, mejorando las características físico - químico del suelo, potencializando el desarrollo, mejora la calidad y aumenta el peso del racimo de banano para exportación, incrementando su rendimiento.

En la presente investigación los bioestimulantes Basf foliar kelp+basfoliar calcio en dosis de $100 \mathrm{cc}+100 \mathrm{cc} / \mathrm{ha}$ dieron los mejores resultados en peso de racimo, largo de dedos y calibra- 
ción de las últimas manos. Donde la longitud de dedos alcanzo $21.59 \mathrm{~cm}$, cuyos resultados coinciden con los de (Aspiazu, 2017) quien en investigación realizada, manifiesta que la aplicación de bioestimulantes Max organic + Biomax 2.0 que presentaron la mayor longitud de dedos con 23,45 cm.

Las respectivas mezclas de los bioestimulantes Basfoliar Kelp + Basfoliar Ca en dosis de 100 $\mathrm{cc}+100 \mathrm{cc} /$ ha nos indica que mejora el rendimiento de banano y mejoramiento en la calidad de la fruta, resultados que coinciden con lo que menciona (Cedeño, 2015) quien además deduce que los bioestimulantes son productos catalogados en la colaboración de los mantenimientos fisiológicos de las plantas, capaces de incrementar el desarrollo, crecimiento de los frutos y un incremento en la producción.

El T2 Basfoliar Kelp + Basfoliar Ca con una dosis de $100 \mathrm{cc}+100 \mathrm{cc} /$ ha nos ayudó a incrementar el respectivo rendimiento del cultivo donde los resultados concuerdan con la argumentación (Mendoza, 2015). quien nos indica que la función principal de los bioestimulantes sucede durante el respectivo proceso metabólico de la planta de banano mediante la fotosíntesis, respiración, nutrición y absorción de nutrientes, actúan en el aumento, el diámetro y altura de pseudotallos, generando más biomasa para obtener una mejor calidad de frutos e incrementos de la producción del cultivo.

\section{REFERENCIAS BIBLIOGRÁFICAS}

Aboboreira, M. (1994). Principales labores del cultivo de banano. Tesis de la finca comercial division banano. Costa Rica

Alban, E. (2014). Evaluacion de la eficacia de citoquinina (cytokin) y un inductor carbonico (carboroot) en tres dosis y en dos epocas en el redimiento de banano de exportacion en una plantacion en produccion variedad gran enana, Canton Quininde de la provincia de Esmerala. Tesis de la Ecuela Superior Politecnica de Chimborazo. Riobamba Ecuador

Aristasabal, M. (2008). Efecto del ácido liberelico y el desmane sobre las características del racimo en plátano. Tesis de la Universidad Nacional de Colombia. Manizaldes - Caldas - Colombia.

\section{CONCLUSIONES Y RECOMENDACIONES}

La aplicación de bioestimulante logró incrementar el rendimiento del cultivo de banano y la calidad de la fruta aplicándolo en el momento que tenía 4 y 10 días de enfunde, y los resultados a saber fueron:

El mejor tratamiento que fue el T2 donde se mezclaron por partes iguales los productos de Basfoliar Kelp + Basfoliar Ca con una dosis de $100 \mathrm{cc}+100 \mathrm{cc} / \mathrm{ha}$ en un litro de agua se pudo observar un mejoramiento significativo de las características agronómicas evaluadas, comparadas con las otras dosis aplicadas y el testigo.

Los resultados del mejor tratamiento que se destacaron en la presente investigación fueron, aumento de peso del racimo con $36.06 \mathrm{~kg} /$ racimo mientras que en el largo de los dedos de la últimas manos se obtuvo un tamaño de 8.5 pulgadas/racimo; en la variable respectiva de la calibración de las últimas manos del racimo fue de 41.95 grados/ racimo. Donde la edad de la cosecha de la fruta se redujo a las 10 semanas.

Se recomienda evaluar la aplicación de bioestimulantes en otras fases del cultivo con otras dosis, añadiendo nuevas tecnologías al cultivo del banano, marinas y calcio a los 4 y 10 días de enfunde en otros ambientes bananeros con el objetivo de mejorar la calidad de la fruta en un menor tiempo de cosecha.

James, C. (2009). Banano, Origen e influencia en la ecomonía ecuatoriana. Guayaquil - Ecuador: Blogs Digital

Pardo, C., \& Novillo, E. (2016). Proceso de control de calidad para el banano de exportacion en finca bananera. Articulo de la Universidad Tecnica de Machala. Machala - El Oro

El Telegrafo, Editorial (2018). El banano encabeza las exportaciones en 2017. Guayaquil - Ecuador: Diario Nacional. 